\title{
Measurement of temperature, fuel concentration and equivalence ratio fields using tracer LIF in IC engine combustion
}

\author{
S. Einecke ${ }^{1}$, C. Schulz ${ }^{1}$, V. Sick ${ }^{2}$ \\ ${ }^{1}$ PCI, Physikalisch-Chemisches Institut, University of Heidelberg, INF 253, 69120 Heidelberg, Germany \\ (Fax: +49-6221/545-050, E-mail: christof.schulz@urz.uni-heidelberg.de) \\ ${ }^{2}$ MEAM, Mechanical Engineering and Applied Mechanics, University of Michigan, 1231 Beal Avenue, Ann Arbor, MI 48109-2121, USA \\ (Fax: +1-734/764-4256, E-mail: vsick@umich.edu)
}

Received: 10 March 2000/Revised version: 19 April 2000/Published online: 16 August 2000 - @ Springer-Verlag 2000

\begin{abstract}
A technique based on planar laser-induced fluorescence of 3-pentanone, for measurements of absolute concentration, temperature and fuel/air equivalence ratios in turbulent, high-pressure combustion systems such as an internal combustion engine is presented. Quasi-simultaneous excitation with $248 \mathrm{~nm}$ and $308 \mathrm{~nm}$ of 3-pentanone that is used as a fluorescence tracer doped to iso-octane, yields pairs of strongly temperature-dependent fluorescence images. Previous investigations have resulted in information on temperature and pressure dependence of absorption cross-sections and fluorescence quantum yields. Using these data the ratio of corresponding fluorescence images can be converted to temperature images. Instantaneous temperature distribution fields in the compression stroke and in the unburned end-gas of an SI engine were measured. The temperature fields obtained from the two-line technique are used to correct the original tracer-LIF images in order to evaluate quantitative fuel distributions in terms of number densities and fuel/air equivalence ratio.
\end{abstract}

PACS: 07.20.Dt; 42.62.Fi; 33.50.Dq

Knowledge of the spatial distribution of temperature and local fuel/air equivalence ratios in the combustion chamber in IC engines prior to ignition is of major interest when modeling engine combustion and modifying combustion chamber geometries. Especially in modern engines with stratified load and systems with exhaust gas recirculation that both strongly influence ignition and flame development, temperature and fuel concentration inhomogeneities are present. For quantitative measurements of fuel vapor concentrations ketones such as 3-pentanone are frequently used because their evaporation properties are similar to those of common model fuels such as iso-octane [1,2]. Furthermore, the influence of collisional quenching mainly by molecular oxygen is much re-

Dedicated to Professor Jürgen Wolfrum on the occasion of his 60th birthday duced compared to aromatic compounds since the lifetime of excited states is controlled by rapid intersystem crossing. 3 -pentanone possesses an absorption feature between 220 and $340 \mathrm{~nm}$ with the peak near $280 \mathrm{~nm}$ at room temperature [3]. The absorption spectrum exhibits a temperature-induced shift towards longer wavelengths of about $10 \mathrm{~nm}$ per increase of $100 \mathrm{~K}$ (Fig. 1). Upon excitation in this region, fluorescence is emitted between 330 and $550 \mathrm{~nm}$, with a spectral distribution almost independent of the absorbed wavelength. This spectral shift of the absorption, albeit undesired for concentration measurements can be used for measuring temperature, for example when 3-pentanone is seeded to non-fluorescing model fuels, as the fluorescence intensity is a function of the absorption coefficient for a given excitation wavelength, and thus, of temperature. After excitation at two different wavelengths the ratio of the fluorescence signal intensities reflects the local temperature. This was first described by Großmann et al. [3] and later applied to temperature measurements using acetone

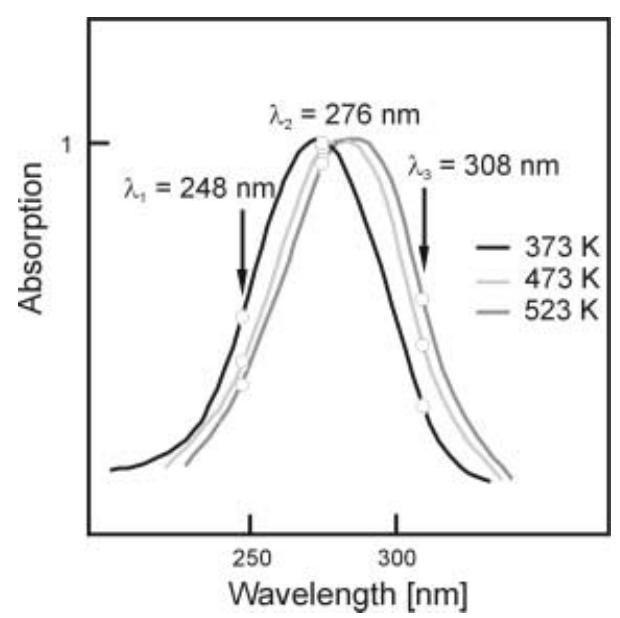

Fig. 1. Temperature shift of the absorption band of 3-pentanone. Wavelengths accessible with excimer laser systems are marked $\left(\lambda_{1}: \operatorname{KrF}, \lambda_{2}\right.$ : Raman-shifted $\mathrm{KrF}$ (first Stokes line in hydrogen), $\left.\lambda_{3}: \mathrm{XeCl}\right)([18])$ 
as tracer [4]. Since this temperature measurement is based on the ratio of signal intensities it is independent of local tracer concentrations and therefore allows measurements of 2D-temperature distributions in non-homogeneously mixed systems.

2D-temperature distributions between 300 and $1000 \mathrm{~K}$ relevant for pre-combustion conditions can hardly be assessed with other laser spectroscopic techniques developed for twodimensional combustion thermometry so far. Rayleigh scattering has been employed for the determination of temperature fields in flames [5]. Engine measurements have been performed as well [6] but problems might occur in realistic engine geometries due to strong elastic scattering off surfaces. Since Rayleigh signal intensities are a function of a speciesdependent scattering cross-section this method is not applicable in non-homogeneously mixed systems where local effective Rayleigh cross-sections are unknown. Kaminski et al. [7] presented the use of a thermometry technique based on two-line LIF-spectroscopy of atomic indium in an internal combustion (IC) engine. This method has been used in static flames by Dec and Keller [8]. However, as the atomic indium is formed in the flame from $\mathrm{InCl}_{3}$ seeded to the fuel, the tracer needs to be activated within the flame front. Therefore, only post-flame gases are accessible to these measurements. With $\mathrm{OH}$ two-line thermometry [9] the measured species is transient and only present in the burned gases close to the flame front. In contrast, NO, doped to fresh gases is present in both, unburned and burned gases. Some uncertainty, however, is induced in the fresh gases by the presence of $\mathrm{NO}_{2}$ formed by reaction of NO with air. With NO-thermometry [10] the considered temperature range can be assessed using 226-nm excitation. Problems due to weak signals and absorption of the short wavelength in engines might occur. A-X $(0,2)$ excitation at $248 \mathrm{~nm}$ on the other hand gives only access to higher temperature ranges for example after the flame front has passed. A combination of both excitation schemes allows the measurement of vibrational temperatures with high sensitivity in the range above $900 \mathrm{~K}$ [11].

When addressing in-cylinder fuel concentration measurements, the temperature-dependent fluorescence cross-section of fuel tracers has been reported as a disadvantage [2]. For single-wavelength excitation, however, the temperature influence on the fluorescence yield can be minimized when the tracer is excited close to its absorption maximum, for example at $276-280 \mathrm{~nm}$ for ketones [12]. This allows a direct measurement of fuel-tracer number densities without the need to know local temperatures. With two-line excitation, in contrast, the measured temperature-sensitive data can then be used to calculate the local temperature and correct for the temperature dependence of the fuel tracer signal obtained for either of the excitation wavelengths. With the simultaneously acquired information about local temperature and fuel number density, local equivalence ratios can be calculated as long as constant oxygen content in the intake air can be assumed.

This paper reports the application of this technique to measurements of temperature distributions and equivalence ratios in a SI engine. Measurements were carried out in an optically accessible two-stroke engine fueled with iso-octane/3-pentanone $(90 / 10 \mathrm{v} / \mathrm{v}) /$ air $[13,14]$. Temperature distribution fields were obtained for numerous detection timings in both, the compression stroke and the power stroke.
After ignition the temperature could still be measured in the unburned gas region using the same technique.

\section{Theoretical background}

The fluorescence signal $S$ for weak laser excitation is given by the following equation:

$S(\lambda, p, c, T)=c I_{\text {Laser }}(\lambda) V N \sigma(\lambda, p, x, T) \phi(\lambda, p, x, T)$,

where $I_{\text {Laser }}$ is the local laser pulse energy in the detection volume $V, N$ is the number density and $\sigma$ the molecular absorption cross section of the marker, and $\phi$ its fluorescence quantum yield. $\sigma$ and $\phi$ depend on the excitation wavelength $\lambda$, pressure $p$, mixture composition $x$, and temperature $T$. The factor $c$ comprises geometrical arrangements and detection optics properties. If the same volume $V$ is excited by two laser pulses of different wavelengths (with a short temporal delay to ensure separate detection of the induced fluorescence), the ratio

$$
\frac{S_{1}\left(\lambda_{1}, p, T\right)}{S_{2}\left(\lambda_{2}, p, T\right)}=\frac{I_{\text {Laser }_{1}}\left(\lambda_{1}\right)}{I_{\text {Laser }_{2}}\left(\lambda_{2}\right)} \frac{\sigma_{1}\left(\lambda_{1}, p, x, T\right)}{\sigma_{2}\left(\lambda_{2}, p, x, T\right)} \frac{\phi_{1}\left(\lambda_{1}, p, x, T\right)}{\phi_{2}\left(\lambda_{2}, p, x, T\right)}
$$

depends on the pressure- and temperature-induced variation in absorption cross-sections and fluorescence quantum yields whereas it is independent of the observed volume $V$, the detected species' number density $N$ and the detection efficiency $c$. Pressure and possible composition effects can be corrected for using data provided by Großmann [3] and Ossler [15] by including a pressure-dependent ratio $\pi_{1} / \pi_{2}$ that includes effects on $\sigma$ and $\phi$. Then, the remaining ratio of both signals normalized to the respective laser energy is a function $F(T)$ of temperature only.

$$
\frac{S_{1}\left(\lambda_{1}, p, T\right) / I_{\text {Laser }_{1}}\left(\lambda_{1}\right) \pi_{2}}{S_{2}\left(\lambda_{2}, p, T\right) / I_{\text {Laser }_{2}}\left(\lambda_{2}\right) \pi_{1}}=\frac{\sigma_{1}\left(\lambda_{1}, T\right) \phi_{1}\left(\lambda_{1}, T\right)}{\sigma_{2}\left(\lambda_{2}, T\right) \phi_{2}\left(\lambda_{2}, T\right)}=F(T) .
$$

A strong temperature dependence of the ratio, hence accuracy, can be expected when $\lambda_{1}$ and $\lambda_{2}$ are selected on opposite sides of the absorption maximum. This results in a temperature gradient with opposite sign and therefore maximizes sensitivity to temperature changes. In our experiments we used excitation at 248 and $308 \mathrm{~nm}(\mathrm{KrF}$ and $\mathrm{XeCl}$ excimer laser): with increasing temperature, the fluorescence intensity decreases after excitation at $248 \mathrm{~nm}$ [3] whereas it strongly increases after excitation at $308 \mathrm{~nm}$ [16]. With a calibration measurement as shown in Fig. 2, the properly corrected ratio of fluorescence signals can then be directly converted into temperature. Figure 2 was composed using data for excitation at $248 \mathrm{~nm}$ from Großmann et al. [3] and for excitation at $308 \mathrm{~nm}$ from Tait and Greenhalgh [16]. The ratio was normalized to unity for a temperature of $373 \mathrm{~K}$. A single calibration measurement to scale the measured fluorescence ratio at a known temperature with the calibration data as displayed in Fig. 2, gives access to the temperature at any given point in a 2D-image, even if the physical properties (i.e. mixture ratio) in the measurement volume are transient. 


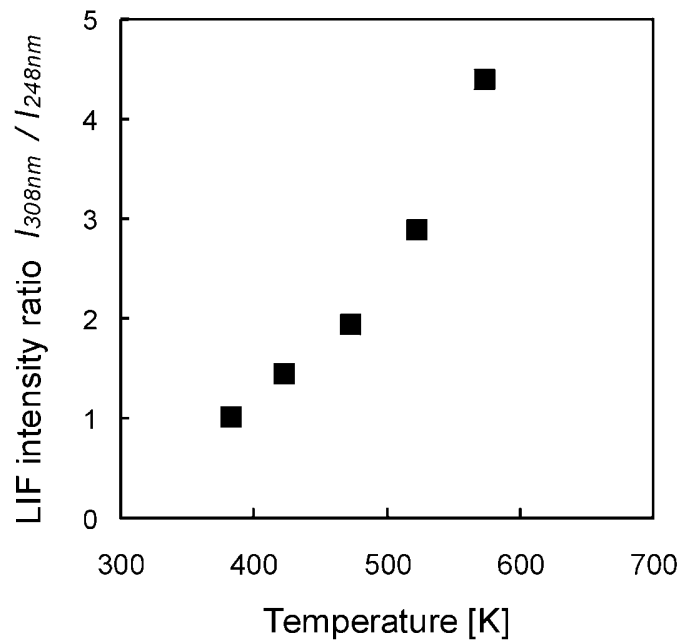

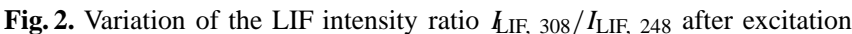
at $308 \mathrm{~nm}$ and $248 \mathrm{~nm}$, respectively, with temperature

Using a hydrogen Raman shift cell pumped by a narrowband $\mathrm{KrF}$ excimer laser allows us to generate two suitable wavelengths (248 and $312 \mathrm{~nm}$ ) with a single laser, which can be used for temperature measurements after optically delaying one of the laser beams to ensure separate signal detection. With the wavelength combination shown here, the maximum temperatures are restricted to approximately $900 \mathrm{~K}$ since signal intensities upon excitation at $248 \mathrm{~nm}$ are getting very weak. Replacing the $\mathrm{KrF}$ excimer laser by a frequencyquadrupled Nd:YAG laser at $266 \mathrm{~nm}$ might overcome this limitation. However, Fig. 5 of [3] shows an increasing absorption feature at short wavelengths for higher temperatures which needs to be addressed more closely in the future.

Having determined the local temperatures now allows us to correct the fluorescence signals to calculate the number density $N$ of tracer molecules:

$N=\frac{S_{i}\left(\lambda_{i}, p, T\right)}{I_{\operatorname{Laser}_{i}}\left(\lambda_{i}\right) \sigma_{i}\left(\lambda_{i}, p, x, T\right) \phi_{i}\left(\lambda_{i}, p, x, T\right) V c}$.

A calibration using a known number density of tracer molecules at a known temperature scales $N$ to absolute number densities. Otherwise the volume $V$ and the constant $c$ would not be accounted for and the analysis would yield relative number density distributions. As the concentration of the tracer in the non-fluorescent fuel is known, the absolute number density of fuel is now determined. Using the ideal gas law the local mole fraction can then be calculated:

$x_{\text {Fuel }}=\frac{N_{\text {Fuel }}}{N_{\text {Total }}}=\frac{N_{\text {Fuel }}}{p V / k T}$,

where $k=$ Boltzmann's constant. The fuel/air ratio can then be determined via

$F / A$ ratio $=\frac{N_{\text {Fuel }}}{N_{\text {Air }}}=\frac{N_{\text {Fuel }}}{N_{\text {Total }}-N_{\text {Fuel }}}$

or in terms of the fuel/air equivalence ratio:

$\phi=\left(\frac{N_{\text {Fuel }}}{N_{\text {Total }}-N_{\text {Fuel }}}\right) /\left(\frac{N_{\text {Fuel }}}{N_{\text {Total }}-N_{\text {Fuel }}}\right)_{\text {stoichiometric }}$.
For the combustion of iso-octane $\left(\frac{N_{\text {Fuel }}}{N_{\text {Total }}-N_{\text {Fuel }}}\right)_{\text {stoichiometric }}=$ 0.0168 , thus

$\phi=\left(\frac{N_{\text {Fuel }}}{N_{\text {Total }}-N_{\text {Fuel }}}\right) / 0.0168$.

\section{Experimental}

The engine measurements (Fig. 3) were conducted in a modified production-line single-cylinder two-stroke engine (ILO L372, bore: $80 \mathrm{~mm}$, stroke: $74 \mathrm{~mm}$, compression ratio: 8.6) [17]. The original cylinder head was replaced by a quartz ring of $4 \mathrm{~mm}$ height to allow for the entrance and exit of the laser sheets, and a cylindrical full-size quartz window on top through which fluorescence could be monitored. A flat aluminum disk replaced the original piston crown with only a small pocket spared for the spark plug, which was mounted in the cylinder wall. The engine was carburetor-fueled with iso-octane (p.a.) doped with 10\% (v/v) 3-pentanone. For the measurements described in this paper the equivalence ratio was kept at $\phi=0.62$ with an ignition timing at $-20^{\circ} \mathrm{ca}$ (crank angle) with respect to TDC (top dead center). Oil cooling at $353 \mathrm{~K}$ was used instead of the original air cooling to achieve stable cylinder-wall temperature conditions. To avoid any fluorescence interference from lubricants, a thin film of molybdenum sulfide grease applied to the cylinder wall and piston bearings replaced the oil usually added to two-stroke fuel. No interfering fluorescence from the grease was detectable. The engine speed was controlled by a dynamometer and set to $1000 \mathrm{rpm}$. Measurements were carried out both without ignition and in a skip-fired mode with four skipped cycles between ignitions. In-cylinder pressure traces were recorded using a pressure transducer (Kistler 6001) and charge amplifier.

Two excimer lasers (Lambda Physik EMG 150 TMSC and EMG 150 EST), operated with $\mathrm{KrF}(248 \mathrm{~nm})$ and $\mathrm{XeCl}$ $(308 \mathrm{~nm})$, respectively, were fired with a fixed delay of $150 \mathrm{~ns}$

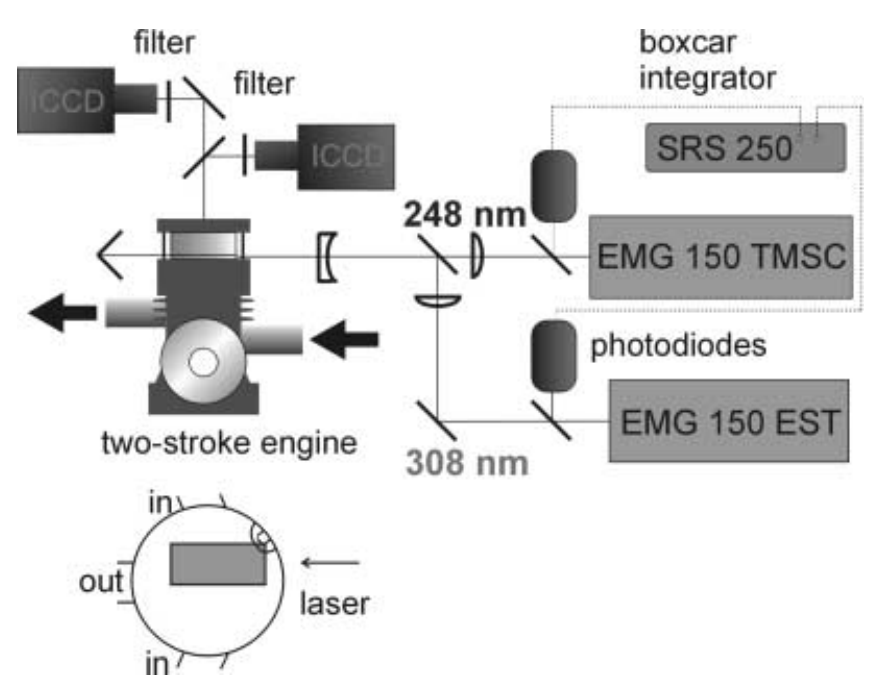

Fig. 3. Experimental setup for the two-line LIF measurements in the twostroke engine. The insert indicates the position of the observed area within the cylinder 
to prevent cross talk of the laser-induced fluorescence signals. Up to $180 \mathrm{~mJ}$ at $248 \mathrm{~nm}$ and $170 \mathrm{~mJ}$ at $308 \mathrm{~nm}$ in pulses of $17 \mathrm{~ns}$ were available at the laser output. An uncoated quartz plate was placed into the beams under $45^{\circ}$ to split off a small amount of energy. This was then measured with a UV-sensitive photodiode for every laser pulse for each laser. The data were acquired along with the pressure data using a Labview-based data acquisition board (National Instruments) and synchronized with the ICCD cameras. To account for the different focal lengths of the cylindrical lenses for $248 \mathrm{~nm}$ and $308 \mathrm{~nm}$ the two laser beams were combined on a dichroic beam splitter (HR $308 \mathrm{~nm}$, uncoated for $248 \mathrm{~nm}$ ) after they each passed through a focusing cylindrical lens $(f=+310 \mathrm{~mm})$ and then were collimated as a combined beam with a diverging cylindrical lens $(f=-40 \mathrm{~mm})$. This way the beam waists could be adjusted individually to ensure perfect overlap at the measurement location in the engine [1]. The dimensions of the two horizontal laser light sheets were measured to approximately $20 \mathrm{~mm}$ width and $0.5 \mathrm{~mm}$ thickness. The laser pulse energies were adjusted to no more than $50 \mathrm{~mJ}$ at the measurement location. This guaranteed that the laser power density at no time exceeded $30 \mathrm{MW} / \mathrm{cm}^{2}$ to ensure excitation in the linear fluorescence regime [15]. The imaged area from the engine was $68 \mathrm{~mm} \times$ $52 \mathrm{~mm}$ onto each of the intensified CCD-cameras (LaVision FlameStar III, $768 \times 572$ pixel), i.e. wider than the width of the light sheets. The signals generated by the two laser pulses were split using a broadband metal-coated beam splitter $(R=31 \%)$ and each part was imaged onto a camera with a $f_{\#}=2, f=100 \mathrm{~mm}$ achromatic UV-lens (Halle). The two cameras were positioned on micrometer-controlled translation and tilt-stages to index the two corresponding images. Any remaining mismatch was corrected during the image processing by translating/rotating one of the images with respect to the other. A millimeter-scaled grid was positioned at the location of the light sheets and photographed with the CCD cameras. Separation of the two images was achieved with the time delay between the laser pulses (150 ns) and a short intensifier gate time $(100 \mathrm{~ns})$. The detection of elastically scattered laser light was suppressed using Schott WG335 filters. The camera signals were digitized with a resolution of 12 bit and stored for data evaluation.

Images taken from engine runs without fuel intake were recorded and subtracted from the individual fluorescence images to account for any spurious unwanted signal due to scattered light. For each laser beam an average spatial intensity distribution was measured by taking fluorescence images of 3-pentanone without running the engine. Ample time was allowed after taking in the 3-pentanone/iso-octane mixture to guarantee a homogenous mixture. These images then served for correction of the two-dimensional fluorescence images with respect to spatial laser energy variations. Additionally, the intensity distributions were scaled with the instantaneous integrated laser energy as measured with the photodiodes.

\section{Results and discussion}

Measurements were performed at a constant engine speed of $1000 \mathrm{rpm}$. The overall equivalence ratio was set to $\phi=0.62$ which was measured with an airflow meter and by monitoring the amount of fuel consumed over a period of time. The low equivalence ratio leads to very slow combustion and thus produced only small pressure increases compared to the motored case (Fig. 4). The setting was chosen to have some of the fuel left for temperature measurements very late in the engine cycle. The acquired fluorescence images were processed with the Cantata suite of image processing utilities from the Khoros 2.1 software package. Individual tracer-LIF signal intensities were corrected for background contribution, incidental laser pulse energy as measured by the photodiodes, and laser sheet inhomogeneities. Pressure effects were taken into account using data from Großmann et al. [3]. By dividing two corresponding corrected images, LIF intensity ratios were obtained and referenced to a calibration measurement at a known temperature. For this calibration the cylinder temperature was stabilized at $353 \mathrm{~K}$ using the engine's oil cooling system while vaporized 3-pentanone was fed into the combustion chamber without operating the engine. Absolute temperatures could then be assigned to each pixel of the resulting images using the calibration curve shown in Fig. 2. The same data could be used to calibrate the fluorescence signals with respect to absolute number densities. Single-shot temperature distributions for various timings in the compression and the power stroke of the fired engine (for location within the engine, see Fig. 3) are shown in Fig. 5. As the flame propagates into the fuel/air mixture, the fluorescence signals disappear and no temperature information can be obtained there. The black regions in the images represent the post-flame gas areas where fuel and fuel tracers are burned. Therefore, the limits of this black area also indicate the instantaneous flame front position. Selected profiles taken along a line in the single-shot images are shown in Fig. 6. The temperature gradient at the flame front is too steep to be resolved with the chosen image magnification. The profiles show that the spatial variation of temperature in the unburned end-gas is quite small, especially during the compression stroke, as could be expected for a skipfired carbureted engine. The over-all temperature is increas-

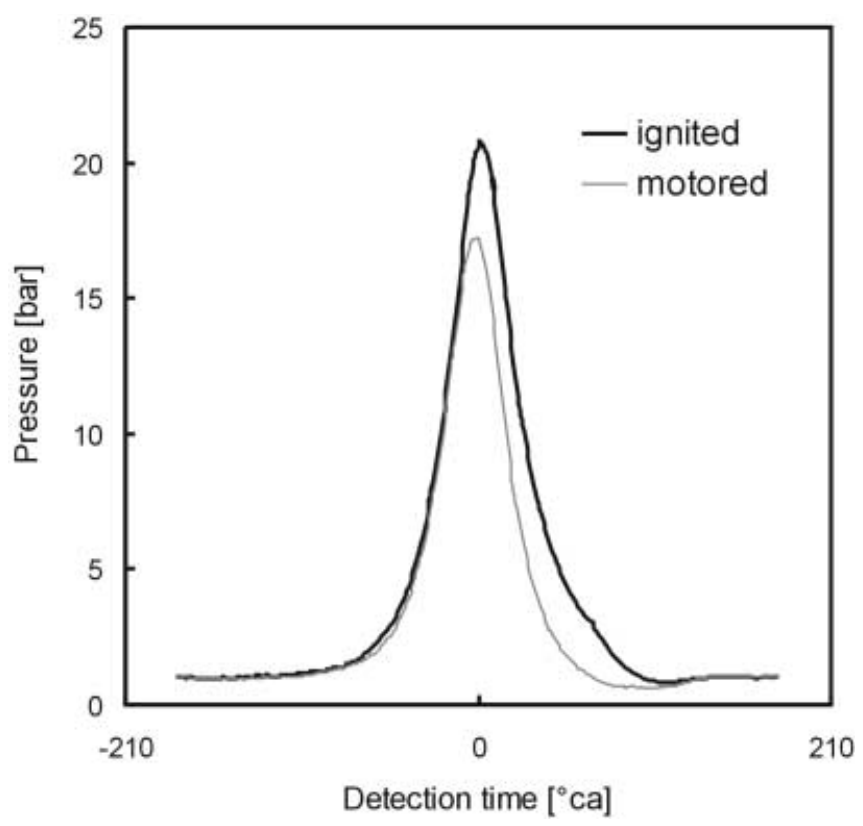

Fig. 4. Pressure trace in the two-stroke engine for a motored and a fired cycle 

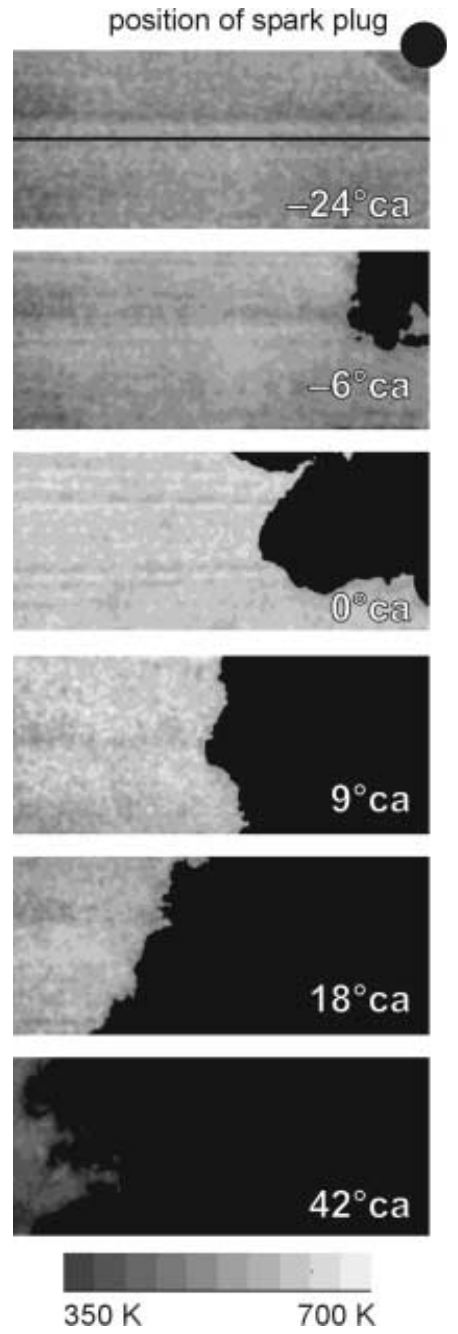

Fig. 5. Single-shot temperature fields for various detection timings. The flame development is visible from the black area where the fluorescent tracers have been burned by the progressing flame. The line in the upper frame indicates the position of the profiles shown in Fig. 6

ing up to top dead center (TDC), followed by a decrease due to the expansion of the gas mixture until $42^{\circ}$ ca when the flame has almost reached the cylinder walls. The observed

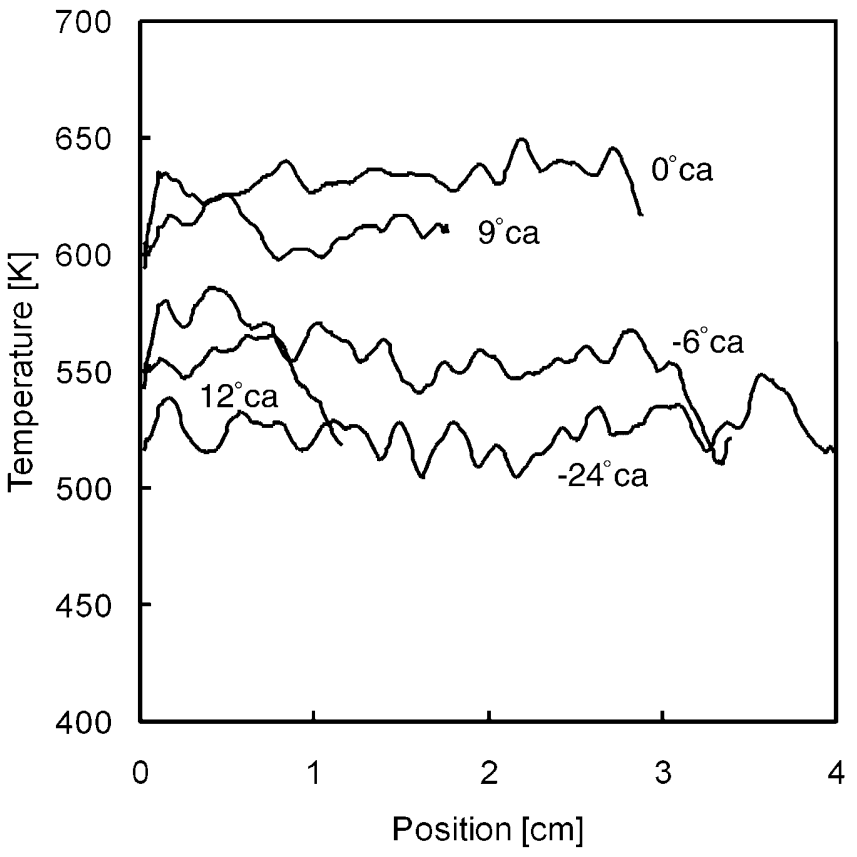

Fig. 6. Temperature profiles obtained from single shot measurements for various detection timings. The position of the line is depicted in Fig. 5

homogeneous temperature distribution justifies the extraction of a smaller region $(10 \mathrm{~mm} \times 7 \mathrm{~mm})$ for the determination of average temperatures. Figure 7 shows some temperature histograms, obtained from 100 single-temperature measurements within these areas. The standard deviation of these data, which includes engine cycle-to-cycle variations as well as the statistical error of the method shows that the temperature measurements can be obtained with a precision of better than $\pm 17 \mathrm{~K}$ in the motored and $\pm 25 \mathrm{~K}$ in the fired engine. This corresponds to a relative error of approximately $4 \%$. Figure 8 displays the average temperature including the above-mentioned errors as a function of crank angle, derived from 100 single measurements per data point. The experimental results are compared to adiabatic calculations assuming a temperature-independent empirical ratio of heat capacities of $\kappa=1.33$. The value $\kappa=1.4$ for pure air is reduced due to the presence of fuels (iso-octane:
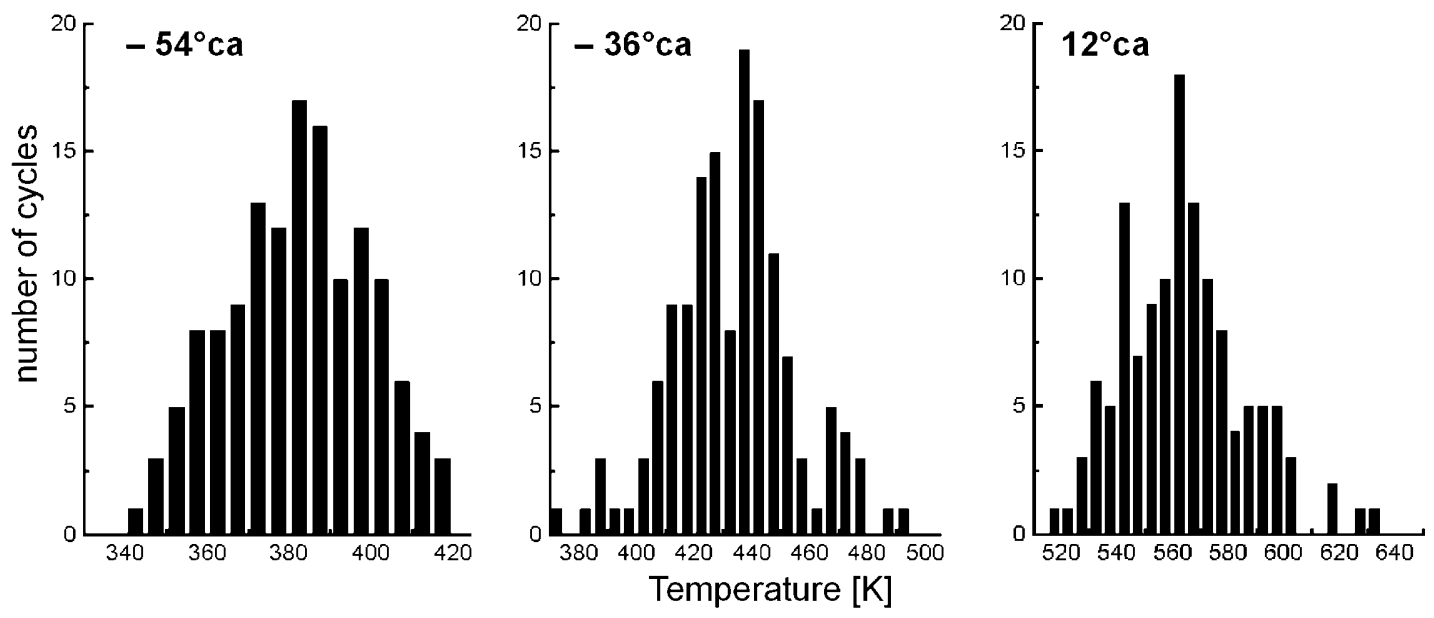

Fig. 7. Histograms for single-shot temperature measurements in the motored engine for various detection timings 


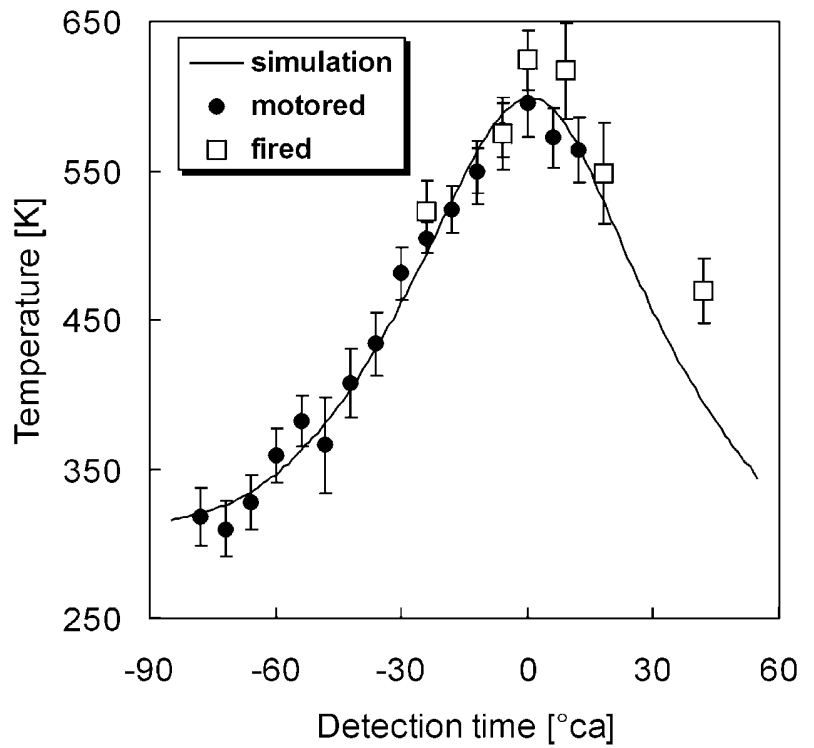

Fig. 8. In-cylinder temperature measurements for the motored and the fired engine as a function of crank angle. The data are compared to a adiabatic calculation for the temperature trace for the motored case

$\kappa=1.04)$. End gas temperatures for engine operation with combustion are higher compared to the motored case. However, variations are rather small due to the minor changes in peak pressures (Fig. 4) as a consequence of the lean fuel/air mixtures.

The two-line tracer-LIF measurement allows further quantification of fuel concentration distributions. Since LIF signal intensities obtained with either excitation wavelength are strongly temperature-dependent, temperature information is required to correct for this effect when quantitative fuel concentration measurements are required. Figure 9 shows the LIF-intensities upon $308 \mathrm{~nm}$ excitation for three singleshot images $\left(18^{\circ} \mathrm{ca}\right)$. According to (4) the absolute number
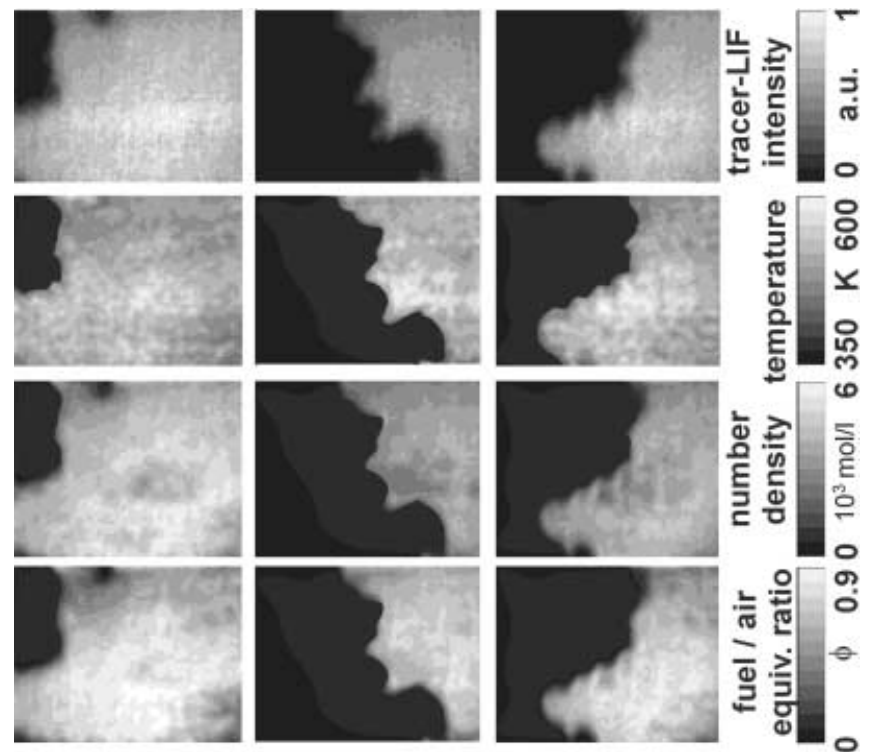

Fig. 9. LIF-intensities upon $308 \mathrm{~nm}$ excitation, temperature fields, tracer number density and fuel/air equivalence ratio distributions in the two-stroke engine. The figure shows three different single-shot images at $18 \mathrm{ca}$ density of fuel molecules can then be determined using the corresponding temperature images shown in the second row. The correction for temperature effects was performed on a per-pixel basis. The corrected LIF images are then calibrated to absolute number densities using the measurements under perfectly mixed conditions where the fuel concentration was calculated from the ratio of measured air flow and fuel consumption. The resulting images give absolute fuel number densities (third row). Since pressure gradients within the cylinder can be safely neglected temperature images allow the calculation of total number densities, which enable the calculation of fuel/air equivalence ratio distributions (fourth row) using (8). This series of images shows how the actual equivalence ratio corresponds to the measured LIF intensity.

\section{Conclusions}

Knowledge of the local fuel/air equivalence ratio $\phi$ is of major importance in engine development. The equivalence ratio determines ignitability, flame propagation, temperature, burnout and formation of pollutants such as nitric oxide, carbon monoxide and unburned hydrocarbons. Controlled spatial variations of $\phi$ are especially crucial for a reliable and clean operation of lean burning engines with stratified load. A measurement technique that is capable of imaging the spatial distribution of $\phi$ would therefore be of great importance for the development of such engines.

Two-line planar LIF-imaging of 3-pentanone, which is added to iso-octane as the primary fuel, provides a technique for measuring the temperature distribution and temperature development in the unburned gas region. The technique was applied to turbulent combustion with inhomogeneous fuel distribution under transient conditions in SI engine combustion. A measurement precision of $4 \%$ was achieved for single-shot temperature images. Temperature distributions obtained from the two-line measurement can subsequently be used to further quantify the fuel distribution as obtained from a single-wavelength measurement. Thus, the temperature influence on tracer LIF-imaging as reported in several applications can be accounted for. Not only can temperature effects on the determination of the number density be accounted for but also the temperature information can be used to calculate local fuel/air equivalence ratios.

Acknowledgements. This work was partly funded by the Commission of the European Community as project 4 Space (Contract No. BRPR-CT970462). Financial support for S. Einecke was granted by the State of Baden-Württemberg and the University of Heidelberg according to the Landesgraduiertenförderungsgesetz (LGFG). The engine was provided by $\mathrm{J}$. Warnatz, and U. Maas, ITV Stuttgart, within an ongoing collaborative research effort. Contribution to the experimental work by Robert Schieß1 is gratefully acknowledged.

\section{References}

1. A. Arnold, A. Buschmann, B. Cousyn, M. Decker, F. Vannobel, V. Sick, J. Wolfrum: SAE Paper 932696 (1993)

2. T. Itoh, A. Kakuho, H. Hishinuma, T. Urushiahara, Y. Takagi, K. Horie, M. Asano, E. Ogata, T. Yamasita: SAE paper No. 952465 (1995)

3. F. Großmann, P. Monkhouse, M. Ridder, V. Sick, J. Wolfrum: Appl. Phys. B 62, 249 (1996) 
4. M.C. Thurber, F. Grisch, R.K. Hanson: Opt. Lett. 22, 251 (1997)

5. A. Buschmann, F. Dinkelacker, T. Schäfer, M. Schäfer, J. Wolfrum: Proc. Combust. Inst. 26, 437 (1996)

6. C. Schulz, V. Sick, J. Wolfrum, V. Drewes, M. Zahn, R. Maly: Proc. Combust. Inst. 26, 2597 (1996)

7. C.F. Kaminski, J. Engström, M. Aldén: Proc. Combust. Inst. 27, 85 (1998)

8. J.E. Dec, J.O. Keller: Proc. Combust. Inst. 21, 1737 (1986)

9. A. Arnold, B. Lange, T. Bouché, T. Heitzmann, G. Schiff, W. Ketterle, P. Monkhouse, J. Wolfrum: Ber. Bunsenges. Phys. Chem. 96, 1388 (1992)

10. J.L. Palmer, B.K. McMillan, R.K. Hanson: Appl. Phys. 63, 167 (1996)

11. W. Bessler, F. Hildenbrand, C. Schulz: In Laser Application to Chemical and Environmental Analysis, Technical Digest (Opt. Soc. of America; Washington DC 2000) p. 149; W.G. Bessler, F. Hildenbrand, C. Schulz: Appl. Opt. (2000) in press
12. H. Krämer, S. Einecke, C. Schulz, V. Sick, S.R. Nattrass, J.S. Kitching: SAE transactions 1998, Vol. 107, pp. 1048-1059, paper No. 982467 (1998)

13. S. Einecke, C. Schulz, V. Sick: In Laser Application to Chemical and Environmental Analysis, Technical Digest (Opt. Soc. of America; Washington DC 1998) p. 84

14. S. Einecke, C. Schulz, V. Sick, A. Dreizler, R. Schieß1, U. Maas: SAE transactions, Vol. 107, pp. 1060-1068, SAE paper No. 982468 (1998)

15. F. Ossler, M. Aldén: Appl. Phys. B 64, 493 (1997)

16. N. Tait, D. Greenhalgh: Ber. Bunsen-Ges. Phys. Chem. 97, 1619 (1993)

17. B. Bäuerle, F. Hoffmann, F. Behrendt, J. Warnatz: Proc. Combust. Inst. 25, 135 (1994)

18. Note: Figures 1-6 are taken either as direct reproduction or with some minor modification from [14]. They are reprinted with permission from SAE paper number 982468 C1998 Society of Automotive Engineers, Inc. 